\title{
Early Detection of Neurodegeneration in Brain Ischemia by Manganese-enhanced MRI
}

\author{
Kevin C. Chan, Ke-xia Cai, Huan-xing Su, Victor K. Hung, Matthew M Cheung, \\ Chi-tat Chiu, Hua Guo, Yang Jian, Sookja K. Chung, Wu-tian Wu and Ed X. Wu
}

\begin{abstract}
This study aims to employ in vivo manganeseenhanced MRI (MEMRI) to detect neurodegenerative changes in two models of brain ischemia, photothrombotic cortical injury (PCI) and transient middle cerebral artery occlusion (MCAO) in rodents. After systemic $\mathrm{Mn}^{2+}$ injection to both ischemic models, a close pattern of T1-weighted hyperintensity was observed throughout different brain regions in comparison to the distribution of GFAP, MnSOD and GS immunoreactivities, whereby conventional MRI could hardly detect such. In addition, the infarct volumes in the posterior parts of the brain had significantly reduced after $\mathrm{Mn}^{2+}$ injection to the MCAO model. It is suggested that exogenous $\mathrm{Mn}^{2+}$ injection may provide enhanced MEMRI detection of oxidative stress and gliosis early after brain ischemia. Manganese may also mediate infarctions at remote brain regions in transient focal cerebral ischemia before delayed secondary damage takes place.
\end{abstract}

\section{INTRODUCTION}

$\mathrm{M}$ ANGANESE (Mn) is an essential constituent of two important metalloproteins involved in the pathophysiology of cerebral hypoxia and ischemia. Mn binds to the mitochondrial Mn-superoxide dismutase (MnSOD) enzyme, which acts against cellular oxidative stress. It also binds to glutamine synthetase (GS), which is a glial specific enzyme for regulating extracellular glutamate and reducing glutamate excitotoxicity. In experimental animal models of stroke, delayed hyperintensity was found in T1-weighted images (T1WI) due to MnSOD accumulation at the ischemic core [1], whereas upon exogenous $\mathrm{Mn}^{2+}$ injection to neonatal rat models of hypoxic-ischemic encephalopathy, upregulation of MnSOD and GS activities

Manuscript received April 7, 2008. This work was supported in part by the Hong Kong Research Grant Council and The University of Hong Kong CRCG grant.

Kevin C. Chan, Ke-xia Cai, Matthew M. Cheung, Chi-tat Chiu, Hua Guo and Jian Yang are with the Laboratory of Biomedical Imaging and Signal Processing and the Department of Electrical and Electronic Engineering, The University of Hong Kong, Pokfulam, Hong Kong (e-mail: kevin_ccw@hkuusa.hku.hk, iinvivo@gmail.com, thew@hkusua.hku.hk, chiuharry@gmail.com,hua.guo@gmail.com, and yj1118@mail.xjtu.edu.cn)

Huan-xing Su, Victor K. Hung, Sookja K. Chung and Wu-tian Wu are with the Department of Anatomy, The University of Hong Kong, Pokfulam, Hong Kong (e-mail: suhx@hkusua.hku.hk, victorh@hkucc.hku.hk, skchung@hkucc.hku.hk, and wtwu@hkucc.hku.hk).

Ed X. Wu is with the Laboratory of Biomedical Imaging and Signal Processing and the Department of Electrical and Electronic Engineering, The University of Hong Kong, Pokfulam, Hong Kong (corresponding author to provide phone: (852) 2819-9713; fax: (852) 2819-9711; e-mail: ewu@eee.hku.hk). was observed, leading to Mn-enhanced MRI (MEMRI) detection of lesions undetectable by other MR modalities [2, 3]. Given a previous study indicating a transient increase of MnSOD in remote brain areas after focal photothrombotic cortical injury (PCI) [4], a relatively non-invasive and reproducible model for stroke, this study aims to employ in vivo MEMRI to detect transient changes in adult rat model of PCI in different brain regions. Further, given the previous reports on the role of manganese as an antioxidant after manganese chloride administration to animals $[5,6]$, we attempted to test the feasibility of mediating remote infarction by delayed administration of manganese after transient middle cerebral artery occlusion (MCAO), in addition to the reproducibility of enhanced MEMRI detection of neurodegeneration in brain ischemia to different strains of rodents.

\section{MATERIALS AND METHODS}

\section{A. Animal Preparation}

Adult Sprague-Dawley rats (200-250 g, N=8) and adult C57BL/6N mice (18-22 g, N=8) were subjected to PCI in the motor cortex center [4] and 30 min-transient MCAO [7], respectively, and were divided into 4 groups ( $\mathrm{N}=4$ each). Two days after surgery, PCI rats in Group 1 and MCAO mice in Group 3 were administered with an intraperitoneal injection of $\mathrm{MnCl}_{2}$ solution $(45 \mathrm{mg} / \mathrm{kg}, 100 \mathrm{mM})$, while the other animals in Groups 2 and 4 received no injection. MRI was performed to the PCI animals at 3, 7, 14 and 21 days after surgery, whereby 2 rats from each group were sacrificed for histology after MR examinations at Day 7 . MEMRI was also performed on 3 additional normal rats before, 1 day and 5 days after $\mathrm{Mn}^{2+}$ injection to compare with the PCI groups at the corresponding time points with and without $\mathrm{Mn}^{2+}$ administration. For MCAO animals, MRI was performed at $3,7,10,14,21$ and 150 days after surgery, whereby 2 mice from each group were sacrificed for histology after MR examinations at Day 10.

\section{B. MRI Protocols}

All MRI measurements were acquired on a $7 \mathrm{~T}$ Bruker scanner. Under inhaled isoflurane anaesthesia (3\% induction and $1.5 \%$ maintenance), the animals were kept warm under circulating water at $37{ }^{\circ} \mathrm{C}$, and were imaged using a receiveonly surface coil for the PCI model, and a mouse brain quadrature resonator for the MCAO model. 2D T1-weighted 
(T1W) RARE sequence was acquired with TR/TE $=400 / 7.5$ $\mathrm{ms}$, RARE factor $=4$, and matrix resolution $=256 \times 256$. For PCI model, FOV $=3.2 \times 3.2 \mathrm{~cm}^{2}$, slice thickness $=1 \mathrm{~mm}$, and NEX $=16$. For MCAO model, FOV $=2.0 \times 2.0 \mathrm{~cm}^{2}$, slice thickness $=0.7 \mathrm{~mm}$, and NEX $=20$. T2-weighted imaging (T2WI) was performed under the same dimensions with TR/TE $=6500 / 120 \mathrm{~ms}$, RARE factor $=12$, and NEX $=$ 2 and 4 respectively for PCI and MCAO. Spin-echo EPI diffusion weighted images (DWI) were acquired with TR/TE $=3000 / 28 \mathrm{~ms}$, matrix resolution $=128 \times 128, \mathrm{NEX}=4, \mathrm{~b}=0$ and $100 \mathrm{~s} / \mathrm{mm}^{2}$, number of shots $=4$ and 30 diffusion directions. $\mathrm{FOV}=3.2 \times 3.2 \mathrm{~cm}^{2}$ for PCI model and $3.0 \times 3.0$ $\mathrm{cm}^{2}$ for MCAO model.

\section{Data Analysis}

The T1W signal intensities at the hypointense ischemic core and peri-infarct areas $0.5 \mathrm{~mm}$ to the core border were measured in both ischemic models using ImageJ v1.38x (Wayne Rasband, NIH, USA). The total brain volumes, and the total infarct volumes indicated by $\mathrm{T} 2 \mathrm{~W}$ hyperintensity were also measured in the ipsilesional side of the brain. Mean values were compared using two-tailed Student's ttests, and the values along the time course were compared using ANOVA. Results were considered to be significantly different when $\mathrm{p}<0.05$.

\section{Histology}

After MR examinations, the animals were transcardially perfused with $4 \%$ paraformaldehyde. The brains were then removed, cut into $10 \mu \mathrm{m}$ sections, and immunostained for glial fibrillary acdic protein (GFAP), manganese superoxide dismutase (MnSOD) and glutamine synthetase (GS), which are markers for gliosis, oxidative stress and glutamate excitotoxicity, respectively.

\section{RESULTS}

\section{A. Enhanced MEMRI detection in PCI model}

When no $\mathrm{Mn}^{2+}$ was applied to the PCI animals in Group 2, the dynamic changes of T2WI and DWI abnormalities generally followed as previously described [8]. At Day 3 after photothrombosis, no T1W signal enhancement was observed in the PCI model at the ipsilesional perilesional rim $(p>0.05)$ as in Figure 1. Yet at Day 7 when the MnSOD activity was supposed to peak in the peri-infarct areas [4], mild T1W signal enhancement was observed at the perilesional rim upon windowing. However, such increase was not statistically significant when compared to the same regions in the normal rats before $\mathrm{Mn}^{2+}$ injection ( $\mathrm{p}>0.05$ ), even though MnSOD and GS immunoreactivities were observed in the ipsilesional cortex as in Figure 2. The apparent $\mathrm{T} 1 \mathrm{~W}$ signals then dropped as time went by.

After $\mathrm{Mn}^{2+}$ injection, T1W hyperintensity was observed at the perilesional rims in all animals. At Day 3 and Day 7 after photothrombosis, animals in Group 1 gave a significant increase in signal intensities at the rims compared to the

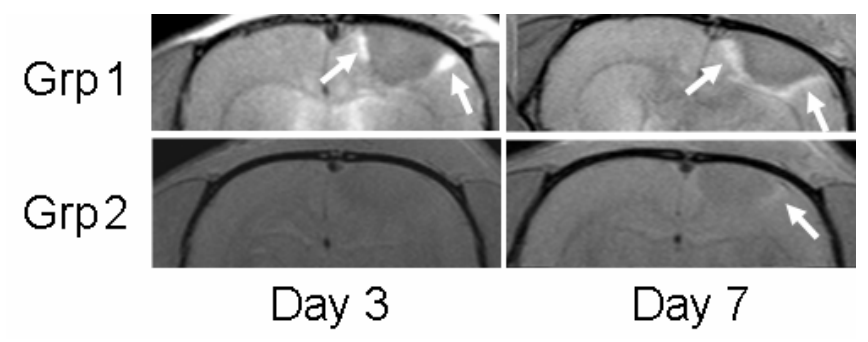

Fig. 1. Typical T1WI at the center of ischemic core at 3 and 7 days after photothrombosis, showing hyperintensity in the perilesional rims (arrows) with (Group 1) and without (Group 2) $\mathrm{Mn}^{2+}$ injection. Hyperintensity was observed in the perilesional rims in Group 2 at Day 7 but was less obvious.

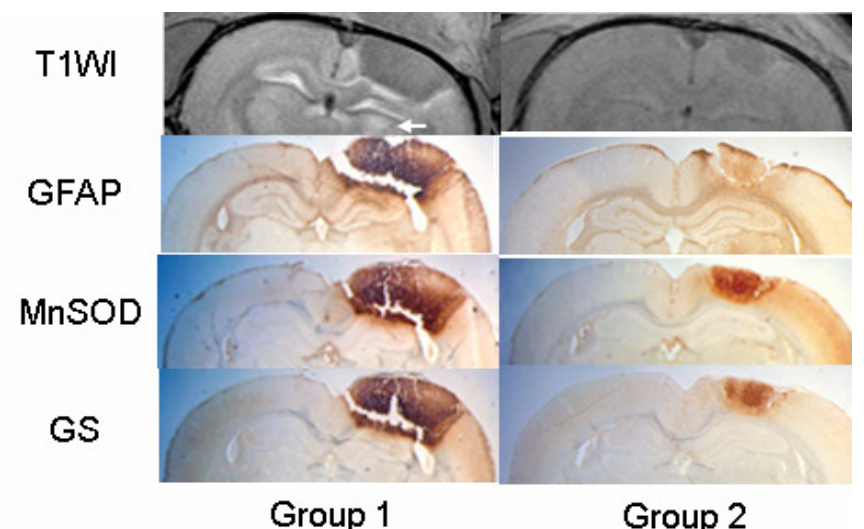

Fig. 2. T1WI at the ischemic core at Day 7 following photothrombosis with (Group 1) and without (Group 2) $\mathrm{Mn}^{2+}$ injection, and the corresponding GFAP, MnSOD and GS immunohistochemical stains at Day 7. Note also the enhancement in the ipsilesional thalamus in Group 1 (arrow).

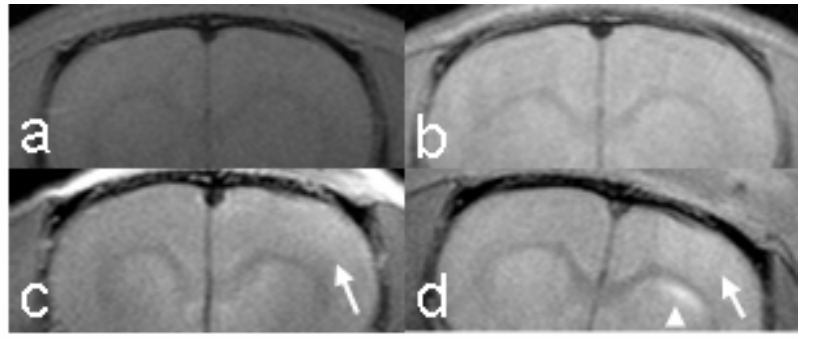

Fig. 3. (Top) Typical T1WI of a normal rat (a) before and (b) 24 hours after $\mathrm{Mn}^{2+}$ injection. (Bottom) Typical T1WI at about $2 \mathrm{~mm}$ anterior to the ischemic core at (c) 3 days and (d) 7 days after photothrombosis, showing hyperintensity in the ipsilesional cortex (arrows) after $\mathrm{Mn}^{2+}$ injection at day 2 in Group 1.
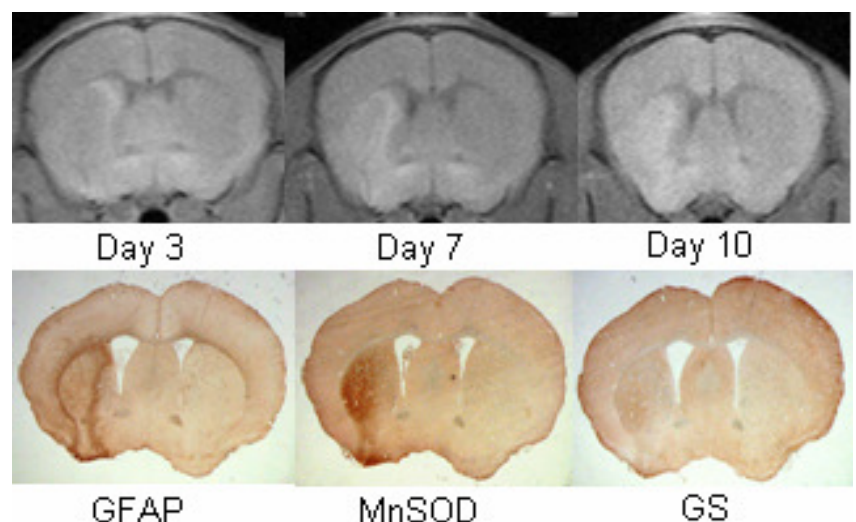

Fig. 4. Mn-enhanced MRI of the striatum at 3, 7 and 10 days following MCAO in Group 3, and the corresponding GFAP, MnSOD and GS immunohistochemical stains at Day 10. 
same location in both the Group 2 animals without $\mathrm{Mn}^{2+}$ injection $(\mathrm{p}<0.05)$, and the normal rats after $\mathrm{Mn}^{2+}$ administration $(p<0.05)$. Enhancement could also be found in the ipsilesional cortex distinct from the ischemic core in Figure 3 and occasionally the subcortical regions in Figures 2 and 3 compared to the normal brains after $\mathrm{Mn}^{2+}$ administration.

\section{B. Enhanced MEMRI detection in MCAO model}

In the ipsilesional dorsolateral striatum, when no $\mathrm{Mn}^{2+}$ was applied in the MCAO mice in Group 4, signal intensity increased in T1WI (ANOVA, $\mathrm{p}<0.01$ ) and decreased in T2WI (ANOVA, $\mathrm{p}<0.01$ ) from Day 3 to Day 21 after MCAO. These were in agreement with previous studies suggestive of delayed ischemic striatal neurodegeneration [1] and inflammatory responses [9]. The diffusion trace values had also dropped in the corresponding hyperintense regions in T2WI, indicative of the presence of oedema. At Day 3, a significant increase in signal intensity at the perilesional rim was observed in T1WI compared to the ischemic core in Group 3 after $\mathrm{Mn}^{2+}$ injection for 24 hours (paired t-test, $\mathrm{p}<0.05)$ but not Group $4(\mathrm{p}=0.18)$, while the Mn-enhanced MRI pattern at Day 10 was in good colocalization with GFAP, MnSOD and GS immunostainings in Figure 4.

\section{Total infarct volumes in PCI and MCAO models}

The infarct volumes indicated by hyperintense regions in T2WI dropped significantly in both models as time went by. The total infracted volumes in ipsilesional forebrain were not different between the two MCAO groups as in Figure 5. However, in the brain regions remote to the ischemic core, $\mathrm{T} 2 \mathrm{~W}$ hyperintensity was observed in the hippocampus, thalamus, midbrain and superior and inferior colliculi in all mice in Group 4, while mild infarction could only be found in the thalamus of one of the mice in Group 3. As shown in Figure 6, total infracted volumes in the posterior parts of the ipsilesional brain had significantly reduced after $\mathrm{Mn}^{2+}$ injection. Note that at 150 days after MCAO, shrinkage of the midbrain and the superior and inferior colliculi was observed in all the 2 remaining animals in Group 4 but not Group 3 as shown in Figure 7. No significant difference was observed in the total infarct volumes between Group 1 and Group 2 in the PCI model.

\section{DISCUSSIONS}

In the present study we demonstrated the early detection of neurodegeneration in brain ischemia by MEMRI in both PCI and MCAO models of different animal strains. These patterns were in good colocalization with GFAP, MnSOD and GS stains and with literature [1,4]. It was found that astroglial cells highly immunostained for GFAP would secrete reactive oxygen species, resulting in an upregulation of MnSOD and GS [2, 10], which are Mn dependent enzymes. In addition, glutamate could be released at the periinfarct areas by increasing the activation of neuronal

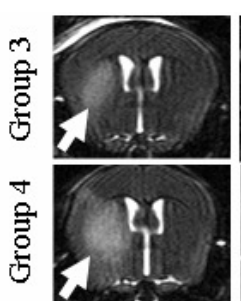

Day 3
Day 7

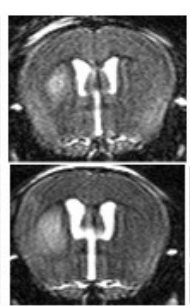

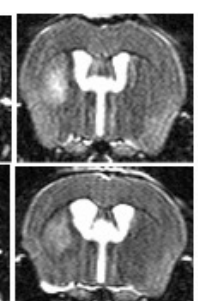

Day 10

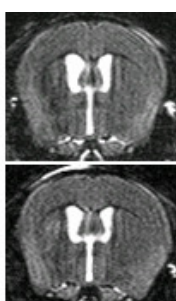

Day 21

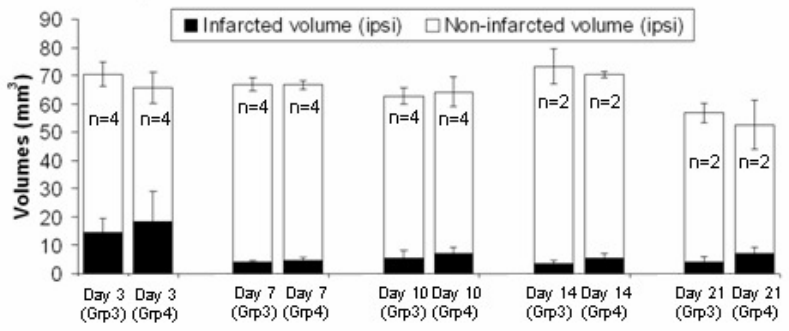

Fig. 5. (Top) Typical T2WI at Bregma $-0.58 \mathrm{~mm}$, showing hyperintensity in the ipsilesional dorsolateral striatum (arrows) with (Group 3) and without (Group 4) $\mathrm{Mn}^{2+}$ injection. (Bottom) Total infarct volumes indicated by $\mathrm{T} 2 \mathrm{~W}$ hyperintensity between Bregma $-1.70 \mathrm{~mm}$ and $1.54 \mathrm{~mm}$ in the ipsilesional forebrain were not different between the groups (unpaired t-test, $\mathrm{p}>0.05$ ).
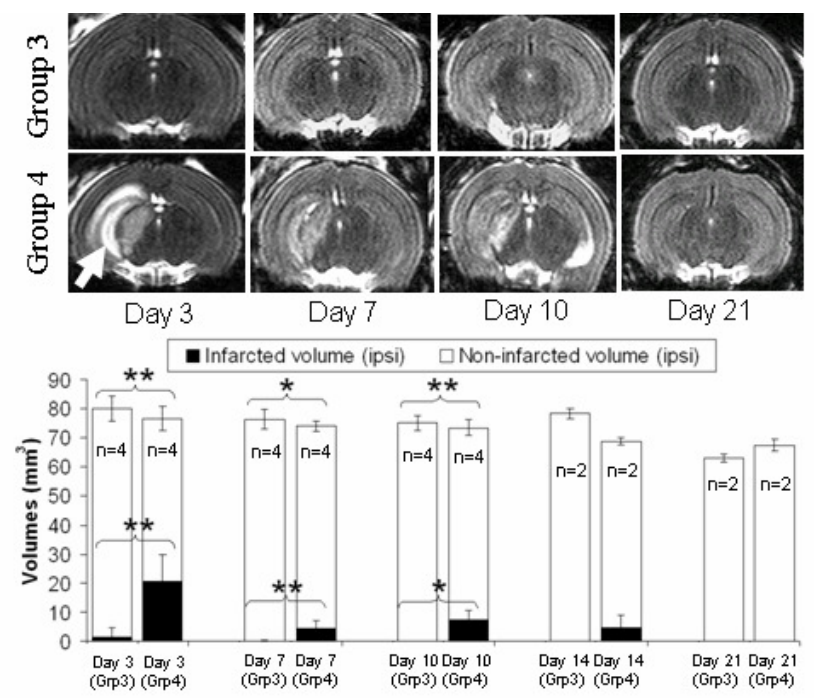

Fig. 6. (Top) Typical T2WI at Begma -3.08, showing hyperintensity in the ipsilesional midbrain and hippocampus (arrow) in Group 4 but not Group 3. (Bottom) Total ipsilesional infarct volumes in the posterior part of the brain between Bregma $-4.96 \mathrm{~mm}$ and $-1.70 \mathrm{~mm}$ had significantly reduced after $\mathrm{Mn}^{2+}$ injection (unpaired t-tests between two groups on infarct volumes (black) or non-infarct volumes (white), ** $\mathrm{p}<0.01, * \mathrm{p}<0.05$ )

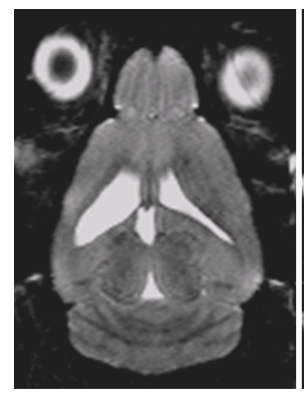

Group 3

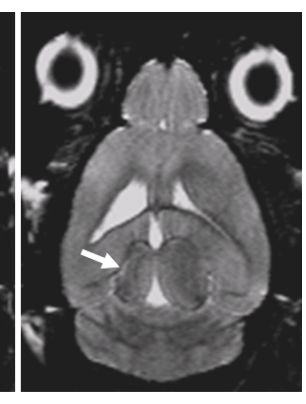

Group 4
Fig. 7. Axial T2WI of mouse brains at 150 days after MCAO. An apparent shrinkage of the ipsilesional midbrain (arrow) was observed in all the 2 animals remained in Group 4 compared to Group 3. 
glutamate receptors which in turn increased membrane permeability to $\mathrm{Ca}^{2+}$ in astrocytes [11-13]. Given $\mathrm{Mn}^{2+}$ has been demonstrated as a calcium analog [14], it is possible that exogenous $\mathrm{Mn}^{2+}$ injection would lead to enhanced MEMRI detection of oxidative stress and gliosis [2, 5]. Note that hyperintense signal areas in MEMRI were shown to match perfectly with areas of microglial activation in the transgenic mouse brains at terminal disease stage [15].

On the other hand, it has been recognized that brain regions remote to the striatum were less susceptible to oxidative stress [16] and might undergo later onsets for delayed neuronal death due to ischemia and reperfusion [17]. Microglial reaction at remote sites such as the neocortex, thalamus and both hippocampi took about 5 days to become fully apparent after transient focal cerebral ischemia [18], whereas brain macrophages were clearly visible at 3-7 days postischemia [19]. Previous MEMRI studies have shown that $\mathrm{Mn}^{2+}$ accumulated and gave peak enhancements in T1WI in the posterior parts of the mouse brain within 24 hours after systemic $\mathrm{Mn}^{2+}$ administration [20]. Given that $\mathrm{Mn}^{2+}$ triggered the scavenging of superoxide and hydroxyl radicals [5], whereby MnSOD and GS activities increased upon $\mathrm{MnCl}_{2}$ administration to normal and ischemic animals [2, 5, $6]$, it is possible that the reduction in infarct volumes in $\mathrm{T} 2 \mathrm{WI}$ in the posterior parts of the MCAO mice might be associated with neuroprotection of $\mathrm{Mn}^{2+}$ at remote sites before apparent delayed secondary damage took place, though further analyses are needed to address this issue. Note also the less neuronal shrinkage in the midbrain areas of the mice in all the 2 remaining animals in Group 3 when compared to those in Group 4 at 5 months after MCAO in Figure 7. Recent studies have shown marked neuroprotective effects of manganese complexes against focal ischemic insults up to 6 hours after ischemia [21, 22]. The results of this study illustrated the potential neuroprotective effects of manganese after administration at a longer postischemic delay. No significant difference was observed in the infarct areas in the forebrain of MCAO mice or the PCI rats between groups with and without $\mathrm{Mn}^{2+}$ injection, possibly due to the ischemic core being readily damaged before $\mathrm{Mn}^{2+}$ administration.

\section{CONCLUSIONS}

Given the distribution of T1W hyperintensity was similar to that of MnSOD and GS immmunoreactivities reported [2, 4], it is likely that exogenous $\mathrm{Mn}^{2+}$ injection may provide enhanced MEMRI detection of oxidative stress and gliosis early in the PCI and MCAO models of different strains. Manganese may also mediate remote brain infartions in transient focal cerebral ischemia before delayed secondary damage takes place.

\section{ACKNOWLEDGMENT}

The authors would like to thank Mr. Dave K. Cheung and
Ms Po-Mak Chan at the Laboratory of Biomedical Imaging and Signal Processing of The University of Hong Kong for their technical assistance.

\section{REFERENCES}

[1] M. Fujioka, T. Taoka, Y. Matsuo, K. Mishima, K. Ogoshi, Y. Kondo, M. Tsuda, M. Fujiwara, T. Asano, T. Sakaki, A. Miyasaki, D. Park, and B. K. Siesjo, "Magnetic resonance imaging shows delayed ischemic striatal neurodegeneration," Ann Neurol, vol. 54, pp. 732-47, Dec 2003.

[2] J. Yang and E. X. Wu, "Detection of cortical gray matter lesion in the late phase of mild hypoxic-ischemic injury by manganese-enhanced MRI," Neuroimage, vol. 39, pp. 669-79, Jan 152008.

[3] J. Yang, P. L. Khong, Y. Wang, A. C. Chu, S. L. Ho, P. T. Cheung, and E. X. Wu, "Manganese-enhanced MRI detection of neurodegeneration in neonatal hypoxicischemic cerebral injury," Magn Reson Med, vol. 59, pp. 1329-39, Jun 2008.

[4] H. J. Bidmon, K. Kato, A. Schleicher, O. W. Witte, and K. Zilles, "Transient increase of manganese-superoxide dismutase in remote brain areas after focal photothrombotic cortical lesion," Stroke, vol. 29, pp. 203-10; discussion 211, Jan 1998.

[5] S. Hussain and S. F. Ali, "Manganese scavenges superoxide and hydroxyl radicals: an in vitro study in rats," Neurosci Lett, vol. 261, pp. 21-4, Feb 121999.

[6] R. K. Singh, K. M. Kooreman, C. F. Babbs, J. F. Fessler, S. C. Salaris, and J. Pham, "Potential use of simple manganese salts as antioxidant drugs in horses," Am J Vet Res, vol. 53, pp. 1822-9, Oct 1992.

[7] A. C. Lo, A. K. Cheung, V. K. Hung, C. M. Yeung, Q. Y. He, J. F. Chiu, S. S. Chung, and S. K. Chung, "Deletion of aldose reductase leads to protection against cerebral ischemic injury," J Cereb Blood Flow Metab, vol. 27, pp. 1496-509, Aug 2007.

[8] M. Schroeter, C. Franke, G. Stoll, and M. Hoehn, "Dynamic changes of magnetic resonance imaging abnormalities in relation to inflammation and glial responses after photothrombotic cerebral infarction in the rat brain," Acta Neuropathol, vol. 101, pp. 114-22, Feb 2001.

[9] S. Wegener, R. Weber, P. Ramos-Cabrer, U. Uhlenkueken, C. Sprenger, D. Wiedermann, A. Villringer, and M. Hoehn, "Temporal profile of T2-weighted MRI distinguishes between pannecrosis and selective neuronal death after transient focal cerebral ischemia in the rat," J Cereb Blood Flow Metab, vol. 26, pp. 38-47, Jan 2006.

[10] E. Pinteaux, M. Perraut, and G. Tholey, "Distribution of mitochondrial manganese superoxide dismutase among rat glial cells in culture," Glia, vol. 22, pp. 408-14, Apr 1998.

[11] I. Suarez, G. Bodega, and B. Fernandez, "Glutamine synthetase in brain: effect of ammonia," Neurochem Int, vol. 41, pp. 123-42, Aug-Sep 2002.

[12] B. K. Siesjo, "Pathophysiology and treatment of focal cerebral ischemia. Part I: Pathophysiology," J Neurosurg, vol. 108, pp. 616-31, Mar 2008.

[13] M. Di Filippo, A. Tozzi, C. Costa, V. Belcastro, M. Tantucci, B. Picconi, and P. Calabresi, "Plasticity and repair in the post-ischemic brain," Neuropharmacology, Feb 132008.

[14] R. G. Pautler, "Biological applications of manganese-enhanced magnetic resonance imaging," Methods Mol Med, vol. 124, pp. 365-86, 2006.

[15] A. Haapanen, U. A. Ramadan, T. Autti, R. Joensuu, and J. Tyynela, "In vivo MRI reveals the dynamics of pathological changes in the brains of cathepsin D-deficient mice and correlates changes in manganese-enhanced MRI with microglial activation," Magn Reson Imaging, vol. 25, pp. 1024-31, Sep 2007.

[16] H. M. Homi, J. J. Freitas, R. Curi, I. T. Velasco, and B. A. Junior, "Changes in superoxide dismutase and catalase activities of rat brain regions during early global transient ischemia/reperfusion," Neurosci Lett, vol. 333, pp. 37-40, Nov 152002.

[17] W. A. Pulsinelli, J. B. Brierley, and F. Plum, "Temporal profile of neuronal damage in a model of transient forebrain ischemia," Ann Neurol, vol. 11, pp. 4918, May 1982.

[18] J. Gehrmann, R. B. Banati, C. Wiessner, K. A. Hossmann, and G. W. Kreutzberg, "Reactive microglia in cerebral ischaemia: an early mediator of tissue damage?," Neuropathol Appl Neurobiol, vol. 21, pp. 277-89, Aug 1995.

[19] C. M. Maier and P. H. Chan, "Role of superoxide dismutases in oxidative damage and neurodegenerative disorders," Neuroscientist, vol. 8, pp. 323-34, Aug 2002.

[20] J. H. Lee, A. C. Silva, H. Merkle, and A. P. Koretsky, "Manganese-enhanced magnetic resonance imaging of mouse brain after systemic administration of $\mathrm{MnCl} 2$ : dose-dependent and temporal evolution of T1 contrast," Magn Reson Med, vol. 53, pp. 640-8, Mar 2005.

[21] G. B. Mackensen, M. Patel, H. Sheng, C. L. Calvi, I. Batinic-Haberle, B. J. Day, L. P. Liang, I. Fridovich, J. D. Crapo, R. D. Pearlstein, and D. S. Warner, "Neuroprotection from delayed postischemic administration of a metalloporphyrin catalytic antioxidant," J Neurosci, vol. 21, pp. 4582-92, Jul 12001.

[22] O. Vajragupta, P. Boonchoong, H. Watanabe, M. Tohda, N. Kummasud, and Y. Sumanont, "Manganese complexes of curcumin and its derivatives: evaluation for the radical scavenging ability and neuroprotective activity," Free Radic Biol Med, vol. 35, pp. 1632-44, Dec 152003. 